\title{
Toxic shock syndrome toxin-I producing Staphylococcus aureus in fulminant necrotizing fasciitis
}

\author{
E Verweij $^{1 *}$, E Kolwijck ${ }^{2}$ and JC Pompe ${ }^{3}$ \\ ${ }^{1}$ Fellow-intensivist, Department of Intensive Care, Radboud University Medical Center, Nijmegen, the Netherlands \\ ${ }^{2}$ Microbiologist, Department of Medical Microbiology, Radboud University Medical Center, Nijmegen, the Netherlands \\ ${ }^{3}$ Intensivist, Department of Intensive Care, Radboud University Medical Center, Nijmegen, the Netherlands
}

\begin{abstract}
We describe a patient presenting with fulminant monomicrobial necrotizing fasciitis (NF) and toxic shock syndrome (TSS) caused by Staphylococcus aureus producing toxic shock syndrome toxin-I (TSST-I). To our knowledge, TSST-I positivity combined with NF has been described only once in previous literature. We discuss the clinical and pathophysiological aspects of both necrotizing fasciitis and toxic shock syndrome and provide a comprehensive overview of causative micro-organisms and the different toxins they produce. We conclude that intravenous immunoglobulin (IVIG) treatment might be considered not only in streptococcal but also in staphylococcal NF.
\end{abstract}

\section{Case}

A 67-year old man was admitted to our Intensive Care Unit (ICU) with septic shock. Five days earlier he presented to the Emergency Department with a mild fever (up to 38 degrees Celsius) and paravertebral back pain. An abdominal ultrasound revealed no pathologic findings (no abdominal aneurysm, gall stones or pancreatitis). His symptoms were attributed to muscular pain possibly caused by influenza (endemic at the time) and he was discharged.

Over the course of 5 days he deteriorated, with severe and progressive back pain with radiation to both legs (which caused an inability to walk) and high fever (up to 41 degrees Celsius). On examination he was tachycardic (heart rate $130 \mathrm{bpm}$ ), hypotensive (initial systolic BP $70 \mathrm{mmHg}, 99 \mathrm{mmHg}$ after $3 \mathrm{~L}$ fluid resuscitation). He was alert with a respiratory rate of $20 / \mathrm{min}$ and oxygen saturation of $97 \%$ on $4 \mathrm{~L} \mathrm{O}_{2} / \mathrm{min}$. Abnormalities on physical examination included basal crepitations, a small pustulous skin lesion on his right upper abdomen and cold extremities with mottled blue skin; no other skin lesions were present, nor were any cardiac murmurs present. Examination of his legs showed no sensory or motor deficits, normal peripheral arterial pulsations and soft, non-tender calves. Laboratory results showed a slight leucopenia $\left(3.8 \times 10^{9} / \mathrm{L}\right)$, thrombocytopenia $\left(59 \times 10^{9} / \mathrm{L}\right)$, C-reactive protein $360 \mathrm{mg} / \mathrm{L}$, creatinine $280 \mu \mathrm{mol} / \mathrm{L}$ and lactate $5.4 \mathrm{mmol} / \mathrm{L}$. Blood cultures were drawn, the patient received antibiotics (ceftriaxone $2000 \mathrm{mg}$ according to local protocol) and was admitted to the ICU for further treatment. On repeated examination he developed a discolouration of the skin on the dorsal side of his left thigh extending to just below the knee. It was blue/purple with surrounding erythema and mildly tender on palpation. A CT-scan was performed, showing induration of skin and subcutaneous tissue in the left thigh. No abdominal or retroperitoneal pathology was detected. Because necrotizing fasciitis was suspected, the patient was started on penicillin, clindamycin, gentamicin, intravenous immunoglobulins (IVIG) and underwent emergency surgical exploration. The subcutaneous tissue and fascia were swollen and excisional biopsies showed extensive presence of gram positive cocci. The diagnosis of necrotizing fasciitis was confirmed. Extensive surgical debridement of skin, subcutaneous tissue and muscle fascia of the dorsum of the left upper leg was performed. The patient developed severe septic shock with multi-organ failure and required high dose norepinephrine, vasopressin and steroid treatment. His condition slightly improved after surgery, antibiotics and IVIG administration. However, soon after the initial stabilisation he developed new skin lesions on his right leg for which he underwent a second surgical procedure. During surgery, similar findings to those in the left leg necessitated another debridement. Upon return to the ICU his condition had deteriorated further, with persistent anuria, severe metabolic acidosis with lactate levels of more than $15 \mathrm{mmol} / \mathrm{L}$ and cardiac failure. At that point, further treatment was considered futile and the patient died immediately after withdrawal of treatment. Methicillin sensitive Staphylococcus aureus was found in blood cultures, urine cultures and tissue cultures. Toxic shock syndrome toxin 1 gene was detected by polymerase chain reaction (PCR) in the Staphylococcus aureus strains isolated. Genes for other staphylococcal toxins (enterotoxin A-D, exfoliative toxin A/B and Panton-Valentine leucocidine) were not present Influenza testing had not been performed.

\section{Background}

Necrotizing fasciitis caused by a single microorganism is reported in the minority of cases. More specifically, necrotizing fasciitis due to Staphylococcus aureus as the only pathogen is not often described in

Correspondence to: Eva Verweij, Fellow-intensivist, Department of Intensive Care, Radboud University Medical Center, Nijmegen, the Netherlands; E-mail: mail@evaverweij.net

Key words: necrotizing fasciitis, staphylococcal toxic shock syndrome, toxic shock syndrome toxin-I, Staphylococcus aureus

Received: November 03, 2015; Accepted: November 30, 2015; Published: December 03, 2015 
literature $[1,2]$. The combination of necrotizing fasciitis together with staphylococcal toxic shock syndrome, as demonstrated by presence of toxic shock syndrome toxin-I (TSST-I), has been published only once previously [3]. We will hereafter discuss both entities.

\section{Necrotizing fasciitis}

Necrotizing fasciitis (NF) is an infectious disease involving subcutaneous tissue and muscle fascia. It is characterised by fever, tenderness, swelling and erythema. Pain disproportionate to the examination findings is frequently described. Other findings that may develop later are numbness of the skin, crepitus and skin discoloration and/or necrosis. These late features are quite specific but absent in more than half the cases $[1,4]$. Diagnosis can be difficult as symptoms may be atypical or absent; a patient may present with profound shock without skin abnormalities. A high index of suspicion is essential. The disease typically has a rapidly progressive course with extension of infection and development of symptoms of systemic toxicity [2].

Necrotizing fasciitis can be divided into categories based on the microbial etiology (Table 1). Four types have been described [1,2,46]. Type $1 \mathrm{NF}$ is polymicrobial, usually with at least one anaerobic bacterium (e.g. Bacteroides, Peptostreptococcus, Fusobacterium, or Clostridium species) in combination with a facultative anaerobic bacterium (usually non-group A streptococci or enterobacteria such as E. coli, Klebsiella or Proteus species). Usually 3 or more microorganisms are involved. When located in the scrotal/perineal area this type of necrotizing fasciitis is known as Fournier gangrene. Risk factors especially apply for type $1 \mathrm{NF}$ and include age of more than 50 years, diabetes mellitus, peripheral vascular disease, HIV, alcohol abuse, and poor nutritional status $[1,7]$.

Type $2 \mathrm{NF}$ is usually monomicrobial and involves group A hemolytical streptococci. However combinations with staphylococci are described $[1,2]$. This type usually affects younger patients without comorbidities. More than in type $1 \mathrm{NF}$ there is a history of trauma or surgery [1].

Type 3 NF is caused by Vibrio vulnificans (particularly associated with chronic liver disease) or Aeromonas species [8]. A fourth type has

Table 1. Necrotizing fasciitis divided into categories based on the microbial etiology [1,4,6$8,10-12]$.

\begin{tabular}{|l|l|c|}
\hline \multirow{3}{*}{$\begin{array}{l}\text { Type I } \\
\text { Polymicrobial }\end{array}$} & Aerobes (gram+) & $40 \%$ \\
\cline { 2 - 3 } & Streptococci & $9-42 \%$ \\
\cline { 2 - 3 } & S. aureus & $5-36 \%$ \\
\cline { 2 - 3 } & Enterococci & $17-\%$ \\
\cline { 2 - 3 } & Aerobes (gram-) & $43 \%$ \\
\cline { 2 - 3 } & E. coli & $7-16 \%$ \\
\cline { 2 - 3 } & Klebsiella & $4-23 \%$ \\
\cline { 2 - 3 } & Pseudomonas & $10-14 \%$ \\
\cline { 2 - 3 } & Anaerobes (Bacteroides, Peptostreptococcus, & $7-18 \%$ \\
\hline Type II & Clostridium) & $22-40 \%$ \\
& Haemolytic streptococci (group A-G) & $13-20 \%$ \\
\hline Type III & Staphylococci & $2-22 \%$ \\
\hline Type IV & E. coli & $50 \% *$ \\
& Aibrio vulnificans & $4-17 \%$ \\
\hline & Aeromonas & $5 \%$ \\
\hline
\end{tabular}

* Population of 61 Taiwanese patients [8]

$\uparrow$ Case reports been described, caused by fungal organisms (Candida, Zygomycetes) $[1,2,5,6,9]$. In fact type 3 and 4 are also monomicrobial and could be considered a subcategory of type 2. Most cases of NF (55-80\%) are polymicrobial with anaerobic presence in approximately $25 \%$ of cases $[1,6,10]$.

The use of imaging studies in diagnosing NF is limited. CT might show fascial thickening, oedema, subcutaneous gas and abscess formation, but these findings are not helpful in differentiating between $\mathrm{NF}$ and other soft tissue infections. It could be useful if other causes for shock are considered but the clinician should be aware that surgery should not be delayed $[2,4]$.

NF is a surgical emergency. Early surgical exploration is cornerstone in both confirming the diagnosis and in management. Macroscopic findings are generally sufficient to confirm the diagnosis and include swollen, non-vital, gray necrotic tissue, lack of bleeding due to thrombosed vessels and foul-smelling ('dishwater') discharge [1]. When macroscopic findings are inconclusive, frozen section procedure is necessary to confirm diagnosis of NF. This is based on histological findings which include necrosis of fascial tissue, polymorphonuclear leucocyte infiltration and thrombosis in arteries and veins of the fascia. Deep fascial biopsy for Gram-staining and cultures will usually reveal the pathogen involved (82\% of cases) [10].

When NF is confirmed, aggressive surgical debridement and fasciotomy should be performed. Re-exploration or second-look surgery is recommended within 24 to 48 hours to assess progression of infection and the need for further debridement. On average, 3-4 operative procedures are needed $[2,10]$.

Treatment further consists of supportive care and empirical broadspectrum antimicrobial treatment, which should cover Gram-positive, Gram-negative and anaerobic pathogens. Clindamycin should be added for both synergism and the benefit of lowering toxin production. This has been proven only in group A streptococcal NF, and failure to add clindamycine to the antimicrobial treatment has shown to increase mortality from $14 \%$ to $60 \%$ in patients with group A streptococcal disease [6].

Coverage of methicillin resistant S. aureus (MRSA) should depend on local incidence [2]. Intravenous immunoglobulin (IVIG) administration is also recommended for neutralising or binding of superantigens and toxins in group A streptococcal disease [2].

Mortality has been shown to vary greatly. Between 1 death per 3 to 5 patients (21\% [10], 25-35\% [1], 29\% [13]) to more varying percentages ranging from low to very high (6-76\% [7]) are found in the literature. A recent review found average rates between 20 and 40\% [2]. Patients either die in an early stage due to refractory shock and/or ARDS or succumb to multiple organ failure later on.

The time between admission and surgery is strongly associated with mortality, with cumulative survival decreasing with increasing time between admission and surgery $[1,2,6,10]$. Mortality risk is further affected by both localisation of the infection and underlying conditions of the patient. Perineal and cervical localisation are associated with a higher mortality [14], and immunocompromised patients have a 2 -fold higher risk of dying in the course of NF [2].

The pathogens involved also influence mortality, although only clostridial and fungal pathogens are consequently described to lead to increased mortality; for group A streptococcal NF data are inconsistent although most describe a better prognosis in type I than in type II NF $[1,6,7]$. 


\section{Toxic shock syndrome}

Toxic shock syndrome (TSS) is an acute systemic illness caused by specific bacterial toxins. These toxins act as superantigens and interact with antigen-presenting cells by binding to class II Major Histocompatibility Complex (MHC) molecules, and with T-cells by binding to specific variable regions on the $\beta$-chain of the T-cell antigen receptor. This induces $\mathrm{T}$-cell activation in up to $5-30 \%$ of the patients $\mathrm{T}$-cells, which is much more than in normal T-cell response (when less than $0,01 \%$ of T-cells are activated $[15,16]$ ). Furthermore the superantigens cause production of cytokines such as TNF- $\alpha$, TNF- $\beta$ and several interleukins. This leads to the development of capillary leak and shock [16-19].

There are several bacteria capable of producing toxic shock causing toxins, with streptococci and staphylococci forming the largest groups. The superantigens causing TSS are known as pyrogenic toxin superantigens and are produced by either streptococci or staphylococci [15].

Streptococci are capable of producing exotoxins (e.g. streptococcal pyrogenic exotoxin (SPE) serotype A produced by group A betahaemolytic streptococci). This results in a syndrome known as streptococcal toxic shock syndrome or toxic shock like syndrome. Other SPE serotypes include B, C, F, G, H and J. Due to massive T cell activation by the superantigen, shock can be rapidly progressive, and this syndrome is associated with mortality rates as high as 50 to 80\% [16-18]. The incidence of TSS in group A streptococcal disease is unclear. Symptoms consistent with TSS in patients with group A streptococcal disease have been reported to occur in up to $30-50 \%$ $[6,18]$ which seems to be an overestimation. For patients with NF, presence of streptococcal TSS increases mortality considerably from less than $40 \%$ to $67 \%$ [6].

Staphylococci also are capable of producing exotoxins. They include TSST-I, Staphylococcal Enterotoxins (SEs) serotype A, B, Cn, D, E, I [18], G and H [19], leucocidin and exfoliative toxins A and B. Most SEs and TSST-I are the superantigens that can cause staphylococcal TSS.

Staphylococcal TSS can be divided into menstrual and nonmenstrual TSS, with the menstrual type being associated with tampon use that facilitates TSST-I production by introducing oxygen [18]. The incidence of menstrual TSS is declining. Nowadays, approximately one third to half of TSS cases are non-menstrual, with the clinical setting varying from postoperative wound infections, mastitis, sinusitis, osteomyelitis, arthritis, burns and post-influenza respiratory infections $[18,20]$. While the majority of $S$. aureus in menstrual TSS (93\%) produces TSST-I, this toxin was present in only $40-63 \%$ of nonmenstrual TSS strains $[21,22]$.

Staphylococcal TSS is characterised by sudden onset of fever, rash, gastrointestinal complaints (nausea, vomiting, diarrhoea and abdominal pain) and capillary leak with hypotension due to vasodilation, possibly leading to multiple organ failure [19]. Case fatality rates for both menstrual and non-menstrual staphylococcal TSS are by far not as high as for streptococcal TSS $[17,18]$. Menstrual TSS (i.e. predominantly TSST-I mediated) disease has a slightly lower mortality rate than non-menstrual TSS. These findings suggest a relatively low $\mathrm{T}$ cell activation potency for TSST-I compared to the other toxins involved in staphylococcal TSS (enterotoxin B and C) and the exotoxins involved in streptococcal TSS [19].

\section{Discussion}

Our patient developed profound septic shock and, despite aggressive surgical and antibiotic treatment and maximum supportive care, died shortly after the diagnosis of NF was made. Staphylococcus aureus was cultured from blood, tissue and urine. No other pathogens were found. Presence of TSST-I was confirmed post-mortem. It is possible he developed staphylococcal TSS as a complication of influenza, but this remains unknown since an influenza PCR was not performed.

Little is known about the incidence of staphylococcal TSS in staphylococcal NF. To our knowledge, only one case report has been previously published that describes a patient with a (monomicrobial) NF and TSST-I mediated staphylococcal TSS [3].

It is reasonable to assume that presence of staphylococcal superantigens and thus staphylococcal TSS worsens prognosis in NF similar to streptococcal NF.

Treatment with IVIG is usually recommended in streptococcal disease. IVIG neutralize the superantigens, decrease cytokine production and have been shown to reduce mortality in streptococcal TSS $[18,19]$.

It has been shown however that IVIG also have activity against TSST-I produced by staphylococci [23]. Patients with staphylococcal TSS might therefore also benefit from treatment with IVIG. Our patient showed a slight improvement after IVIG and might be an example of this.

\section{Conclusion}

We report the case of a 67-year-old male who died of profound shock and multi organ failure due to necrotizing fasciitis in combination with TSST-I mediated staphylococcal toxic shock syndrome. This has only been described once previously in literature. Both NF and TSS are known for progressing rapidly; high clinical suspicion index and aggressive surgical management in combination with antibiotic treatment and supportive care are essential. Given the mechanisms of action it is reasonable to assume IVIG have a role in the treatment of patients with staphylococcal TSS. With the rising incidence of MRSA related infections $[1,6,11,24]$ the role of IVIG might turn out to be even more important in controlling these infections.

\section{References}

1. Sarani B, Strong M, Pascual J, Schwab CW (2009) Necrotizing fasciitis: current concepts and review of the literature. J Am Coll Surg 208: 279-288. [Crossref]

2. Hakkarainen TW, Kopari NM, Pham TN, Evans HL (2014) Necrotizing soft tissue infections: review and current concepts in treatment, systems of care, and outcomes. Curr Probl Surg 51: 344-362. [Crossref]

3. Omland LH, Rasmussen SW, Hvolris J, Friis-Møller A (2007) Necrotizing fasciitis caused by TSST-1 producing penicillin-sensitive Staphylococcus aureus--a case report. Acta Orthop 78: 296-297. [Crossref]

4. Anaya DA, Dellinger EP (2007) Necrotizing soft-tissue infection: diagnosis and management. Clin Infect Dis 44: 705-710. [Crossref]

5. Giuliano A, Lewis F Jr, Hadley K, Blaisdell FW (1977) Bacteriology of necrotizing fasciitis. Am J Surg 134: 52-57. [Crossref]

6. Morgan MS (2010) Diagnosis and management of necrotising fasciitis: a multiparametric approach. J Hosp Infect 75: 249-257. [Crossref]

7. McHenry CR, Piotrowski JJ, Petrinic D, Malangoni MA (1995) Determinants of mortality for necrotizing soft-tissue infections. Ann Surg 221: 558-563. [Crossref]

8. Lee CY1, Kuo LT, Peng KT, Hsu WH, Huang TW, et al. (2011) Prognostic factors and monomicrobial necrotizing fasciitis: gram-positive versus gram-negative pathogens. BMC Infect Dis 11: 5. [Crossref] 
9. Jain D, Kumar Y, Vasishta RK, Rajesh L, Pattari SK, et al. (2006) Zygomycotic necrotizing fasciitis in immunocompetent patients: a series of 18 cases. Mod Pathol 19: 1221-1226. [Crossref]

10. Wong CH, Chang HC, Pasupathy S, Khin LW, Tan JL, et al. (2003) Necrotizing Fasciitis: Clinical Presentation, Microbiology and Determinants of Mortality. J Bone Joint Surg Am 85A: 1454-1460. [Crossref]

11. Tsitsilonis S, Druschel C, Wichlas F, Haas NP, Schwabe P, et al. (2013) Necrotizing fasciitis: is the bacterial spectrum changing? Langenbecks Arch Surg 398: 153-159. [Crossref]

12. Yahav D, Duskin-Bitan H, Eliakim-Raz N, Ben-Zvi H, Shaked H, et al. (2014) Monomicrobial necrotizing fasciitis in a single center: the emergence of Gram-negative bacteria as a common pathogen. Int J Infect Dis 13-16. [Crossref]

13. Waldron C, Solon JG, O'Gorman J, Humphreys H, Burke JP, et al. (2015) Necrotizing fasciitis: The need for urgent surgical intervention and the impact of intravenous drug use. Surgeon 13: 194-199. [Crossref]

14. Elliott DC, Kufera JA, Myers RA (1996) Necrotizing soft tissue infections. Risk factors for mortality and strategies for management. Ann Surg 224: 672-683. [Crossref]

15. Dinges MM, Orwin PM, Schlievert PM (2000) Exotoxins of Staphylococcus aureus. Clin Microbiol Rev 13: 16-34. [Crossref]

16. Llewelyn M, Cohen J (2002) Superantigens: microbial agents that corrupt immunity. Lancet Infect Dis 2: 156-162. [Crossref]
17. Shalaby T, Anandappa S, Pocock NJ, Keough A, Turner A (2014) Lesson of the month 2: toxic shock syndrome. Clin Med 14: 316-318. [Crossref]

18. Low DE (2013) Toxic shock syndrome: major advances in pathogenesis, but not treatment. Crit Care Clin 29: 651-675. [Crossref]

19. McCormick JK1, Yarwood JM, Schlievert PM (2001) Toxic shock syndrome and bacterial superantigens: an update. Annu Rev Microbiol 55: 77-104. [Crossref]

20. Hajjeh RA, Reingold A, Weil A, Shutt K, Schuchat A, et al. (1999) Toxic shock syndrome in the United States: surveillance update, 1979 1996. Emerg Infect Dis 5: 807-810. [Crossref]

21. Garbe PL, Arko RG, Reingold AL, Graves LM, Hayes PS, et a.1 (1985) Staphylococcus aureus isolates from patients with nonmenstrual toxic shock syndrome. Evidence for additional toxins. JAMA 253: 2538-2542. [Crossref]

22. Schlievert PM (1986) Staphylococcal enterotoxin B and toxic-shock syndrome toxinare significantly associated with non-menstrual TSS. Lancet 1: 1149-1150. [Crossref]

23. Nakae T, Hirayama F, Hashimoto M (2002) Neutralizing activity of human immunoglobulin preparation against toxic shock syndrome toxin-1 [abstract, article in Japanese]. Kansenshogaku Zasshi 76:195-202. [Crossref]

24. Young LM, Price CS (2008) Community-acquired Methicillin-resistant Staphylococcus Aureus emerging as an important cause of necrotizing fasciitis. Surg Infect 9: 469-474. [Crossref]

Copyright: $(02015$ Verweij E. This is an open-access article distributed under the terms of the Creative Commons Attribution License, which permits unrestricted use, distribution, and reproduction in any medium, provided the original author and source are credited. 